\title{
Evaluation of the performance of a motion capture system for small displacement recording and a discussion for its application potential in bone deformation in vivo measurements
}

Proc IMechE Part H:

$J$ Engineering in Medicine 226(II) 838-847 (c) IMechE 2012

Reprints and permissions: sagepub.co.uk/journalsPermissions.nav DOI: 10.1177/0954411912452994 pih.sagepub.com (SAGE

\author{
Peng-Fei Yang ${ }^{1,2,3}$, Maximilian Sanno², \\ Gert-Peter Brüggemann ${ }^{2}$ and Jörn Rittweger ${ }^{1,4}$
}

\begin{abstract}
The aim of this study is to evaluate the performance of a motion capture system and discuss the application potential of the proposed system in in vivo bone-segment deformation measurements. In this study, the effects of the calibration procedure, camera distance and marker size on the accuracy and precision of the motion capture system have been investigated by comparing the captured movement of the markers with reference movement. The results indicated that the system resolution is at least $20 \mu \mathrm{m}$ in a capture volume of $400 \times 300 \times 300 \mathrm{~mm}^{3}$, which mostly covers the range of motion of the tibia during the stance phase of one gait cycle. Within this volume, the system accuracy and precision decreased following the increase of camera distance along the optical axis of the cameras. With the best configuration, the absolute error and precision for the range of $20 \mu \mathrm{m}$ displacement were $1.2-1.8 \mu \mathrm{m}$ and $1.5-2.5 \mu \mathrm{m}$, respectively. Small markers $(\varnothing 3-8 \mathrm{~mm})$ yielded better accuracy and repeatability than the larger marker $(\varnothing 10.5 \mathrm{~mm})$. We conclude that the proposed system is capable of recording minor displacements in a relative large volume.
\end{abstract}

\section{Keywords}

Motion capture system, bone strain, in vivo, accuracy, precision

Date received: 2I August 20II; accepted: 5 June 2012

\section{Introduction}

Bone strain is widely accepted as one of the most important factors in bone adaptation. Reliable and quantitative evidence from many studies over the past few decades suggests an inseparable relationship between bone strain and osteogenic responses. ${ }^{1-4}$ Nevertheless, in vivo bone strain measurement is still technically challenging, especially in humans. Despite the invasiveness, in previous in vivo bone strain studies, the inherent shortcomings of the methodologies based on strain gages in vivo are likely to result in the underestimation of the strain value owing to the low bonding quality. ${ }^{5-8}$ On the other hand, strain gages can only reflect strains in specific areas that are covered by the gages, but yield no information regarding the bonesegment deformation on a large scale. Even more importantly, it is difficult, not to say impossible, to assess bending and torsional strains with strain gages in humans. ${ }^{5}$

In order to overcome the drawbacks of this traditional method, several new methods have been developed to assess bone strains. such as a non-contact

\footnotetext{
'Institute of Aerospace Medicine, German Aerospace Center, Germany ${ }^{2}$ Institute of Biomechanics and Orthopaedics, German Sport University Cologne, Germany

${ }^{3}$ Key Laboratory for Space Bioscience and Biotechnology, Northwestern Polytechnical University, China

${ }^{4}$ Institute for Biomedical Research into Human Movement and Health, Manchester Metropoliation University, UK
}

\section{Corresponding author:}

Peng-Fei Yang, Division of Space Physiology, Institute of Aerospace Medicine, German Aerospace Center, Linder Höhe, D-5I 147, Cologne, Germany.

Email: Pengfei.Yang@dlr.de 
optical technique, referred to as digital image correlation (DIC), ${ }^{9-11}$ as well as ultrasound wave propagation. ${ }^{12}$ However, the potential of these methods for in vivo application is still debatable.

Camera-based motion capture systems are nowadays widely used in biomechanics studies. The human motion can be captured by tracking markers that are attached on the skin of the human body. The data processing then involves (a) location of the markers in the two-dimensional (2D)-space of each camera, and (b) reconstruction of each marker in the real-world three-dimensional (3D)-space by convergence of the information from the set of cameras. ${ }^{13}$ Although the performance of the motion capture system varies with the specific system set-up, ${ }^{14}$ it is theoretically possible to achieve very high accuracy with a proper calibration procedure, choice of markers and the positioning of the cameras. Liu et al. ${ }^{15}$ evaluated the accuracy and precision of the Qualisys motion capture system (Qualisys, Inc., Gothenberg, Sweden) in the measurements of small displacements in a small field of view. Results indicated that, in a $68 \times 51 \mathrm{~mm}^{2}$ field of view, the system resolution was $10 \mu \mathrm{m}$. In the range of $20-200 \mu \mathrm{m}$, the absolute displacement errors were from \pm 2.1 to $\pm 3.0 \mu \mathrm{m}$ for diamond markers, and from \pm 2.5 to $\pm 4.25 \mu \mathrm{m}$ when sphere markers were used. The standard deviation of the recording was $1.7-2.3 \mu \mathrm{m}$ for diamond markers, and 1.9-3.9 $\mu \mathrm{m}$ for sphere markers, respectively. ${ }^{15}$ With the Vicon-460 motion capture system (Vicon Motion System Ltd, LA, USA), accuracy of $63 \mu \mathrm{m}$ and precision of $15 \mu \mathrm{m}$ can be achieved in a volume of $180 \times 180 \times 150 \mathrm{~mm}^{3} .{ }^{14}$ Overall, the previous studies have demonstrated that, with proper parameters and set-up, commercially available motion capture systems are able to provide sufficient accuracy and precision (also called repeatability or reproducibility) to measure small movement of the markers in a relatively small 3D volume. If these markers are affixed to the bone, e.g. human tibia, theoretically, the tibia-segment deformation should be able to be measured by capturing the relative movement between the markers. By doing so, the complex measurements of different types of tibia-segment deformation essentially turn out to be the simple measurements of the markers' coordinate in a 3D volume. During the stance phase of one human gait cycle, the range of motion of the tibia is almost constant and limited within a certain $3 \mathrm{D}$ volume. In another words, the movement of the markers that affixed to the tibia during the stance phase of one gait cycle also stay within a certain $3 \mathrm{D}$ volume. As long as the markers' coordinates in this 3D volume is recorded with high accuracy and repeatability, axial, shear and torsion bone-segment deformation should be able to be calculated. However, despite the requirement of high accuracy and repeatability, the very limited volume covered in the aforementioned studies seems to impede the application of motion capture camera systems for in vivo bone-segment deformation measurements. To our knowledge, neither the performance of motion capture systems in a relatively large volume, nor the potential of applying a motion capture system in bone deformation (most commonly refer to tibia) in vivo measurements, has been reported and discussed before.

Taking the human tibia as an example, assuming the distance between the markers affixed in the tibia as $L=20 \mathrm{~cm}$ and the detection limit of the optical method as $100 \mu \varepsilon$ (compared with approximately $2000 \mu \varepsilon$ in vivo tibia strain, ${ }^{6,16}$ the detection limit of $100 \mu \varepsilon$ is acceptable), respectively, the required resolution of the optical method, $\Delta L$, can be calculated as $\Delta L=L \times 100 \mu \varepsilon=20 \mu \mathrm{m}$. Although this is only a rough figure, it can inform us about the required resolution of an optical method to assess the in vivo tibia deformation. We therefore ventured to evaluate the performance of a motion capture system on small displacement measurements $(20 \mu \mathrm{m})$ in a relatively large volume. More specifically we are addressing, first, whether $20 \mu \mathrm{m}$ of resolution is able to be achieved with proposed system configuration in this study was assessed; and second, the accuracy and precision during the measurement of $20 \mu \mathrm{m}$ movement in a large volume were evaluated. In addition, the application potential of this method in bone-segment deformation in vivo measurements was discussed.

\section{Materials and methods}

The Vicon MX motion capture system (Vicon Motion System Ltd, LA, USA), including Vicon F40 cameras (4 Megapixel, $370 \mathrm{fps}$ full frame top speed), MX Giganet controlling hardware module and Vicon Nexus 1.6.1 software, was adopted to capture the motion of the retro-reflective markers. The performance (accuracy and precision) of this system was evaluated to establish the proposed method.

As mentioned above, the markers affixed to the tibia during the stance phase of one gait cycle stays within a certain volume, which is able to be assumed by the length of tibia. Typically, the length of the tibia is approximately $400 \mathrm{~mm}$. Accordingly, the volume of interest during a tibia's stance phase has dimensions of $400 \times 300 \times 300 \mathrm{~mm}^{3}$. In this volume, the performance of this optical system was assessed. Assuming an intermarker distance of $20 \mathrm{~cm}$, one has to resolve marker displacement by $20 \mu \mathrm{m}$ in order to assess strains of $100 \mu \varepsilon$ - a goal that seems worthwhile to achieve. Several previous in vivo bone strain studies with strain gages suggested that more than $2000 \mu \varepsilon$ of bone deformation can be reached during different exercises. ${ }^{5}$ Comparably, we believe that the detection limit of $100 \mu \varepsilon$ in the proposed method would be acceptable for in vivo bone strains recording in humans.

Within the selected volume, the minute movement of the markers was performed by a universal milling machine (DMU 50M, DMG Vertriebs und Service $\mathrm{GmbH}$, Bielefeld, Germany). The milling machine is capable of positioning its spindle in three dimensions 


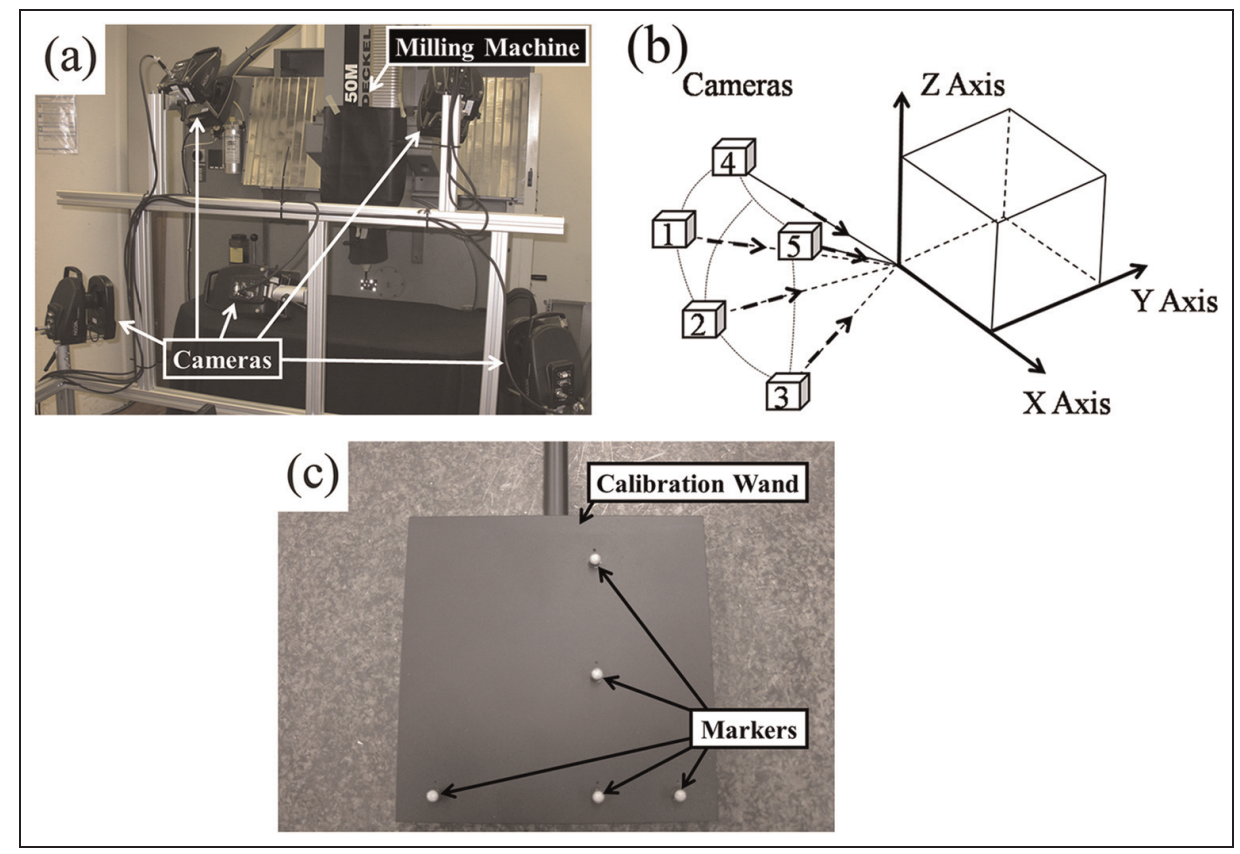

Figure I. The configuration of optical system and calibration procedure. (a) Configuration of the cameras and the milling machine. (b) The sketch of camera distribution and the volume. Boxes I-5 refer to different cameras; dashed arrow: optical axes of cameras; $X, Y$ and $Z$ axes are the three orthogonal directions along which the markers were moved. (c) Custom made calibration wand that was used in this study.

accurately (resolution in all axes: $1 \mu \mathrm{m}$ ). After being affixed on the spindle of the milling machine, the markers were moved along three orthogonal directions within the volume of $400 \times 300 \times 300 \mathrm{~mm}^{3}$ (Figure 1).

\section{Parameters in the optical system configuration}

Cameras setup. Five Vicon F40 cameras with $16 \mathrm{~mm}$ focal length lenses were used in this study. Custommade aluminum frames were used to position the cameras. The cameras were placed as close as possible to the milling machine in order to maximize the resolution of the system. The distance between the cameras and the center of the volume ranged between 100 and $120 \mathrm{~cm}$.

The cameras were set up in near umbrella configuration according to the recommendations by the manufacturer. ${ }^{17}$ The optical axis and location of each camera were adjusted to involve the volume of $400 \times 300 \times$ $300 \mathrm{~mm}^{3}$ into the overlap field of view. The sampling frequency of the system is set at $100 \mathrm{~Hz}$.

System calibration. A custom-made calibration wand (Figure 1(c)), with $\emptyset 4 \mathrm{~mm}$ sphere markers, was used to calibrate the system before the measurements. In the selected volume, manual and automatic calibrations were carried out respectively to assess the effects of the calibration procedure on the performance of this optical system.

The manual calibration was performed by hand according to the manufactures' recommendations. During the automatic full volume calibration, the calibration wand was moved (speed: $5 \mathrm{~m} / \mathrm{min}$ ) along the customprogrammed motion path through the entire volume.
Camera distance and axis. The sphere markers were driven to a total of 80 positions that uniformly distributed in the selected volume (Figure 2). At each position, the markers were driven for a distance of $20 \mu \mathrm{m}$ along the positive direction of three orthogonal axes respectively and back (Figure 2, $X, Y$ and $Z$ axis). For the distance of $20 \mu \mathrm{m}$, the starting position, movement path and the final position of the markers has been recorded. Measurements were repeated three times for each specific condition. The increase of camera distance relative to camera position (Figure 2, D1: the closest distance; D2, D3 and D4: the furthest distance) mostly aligned with $Y$ axis. Twenty positions were located at each distance.

Marker size. In order to assess the effects of marker size on the performance of the proposed optical method, sphere markers in four different sizes (diameter: $\varnothing 3 \mathrm{~mm}, \varnothing 4 \mathrm{~mm}, \varnothing 8 \mathrm{~mm}$ and $\varnothing 10.5 \mathrm{~mm}$ ) were used in this study. The sphere markers were manufactured as copper balls coated with standard Vicon retro-reflective tape (Vicon Motion System Ltd, LA, USA). The smallest markers that are possibly manufactured with a smooth surface $(\emptyset 3 \mathrm{~mm}$ and $\emptyset 4 \mathrm{~mm}$ markers) were selected to evaluate the effects of small markers on the performance of the system. Reasonably large markers ( $\varnothing 8 \mathrm{~mm}$ and $\emptyset 10.5 \mathrm{~mm}$ markers), which are suitable for future in vivo tibia-segment deformation measurements and have low opportunities of markers overlap, were selected as well.

Random noise. Random noise of the system induced by ambient factors has to be considered during very 


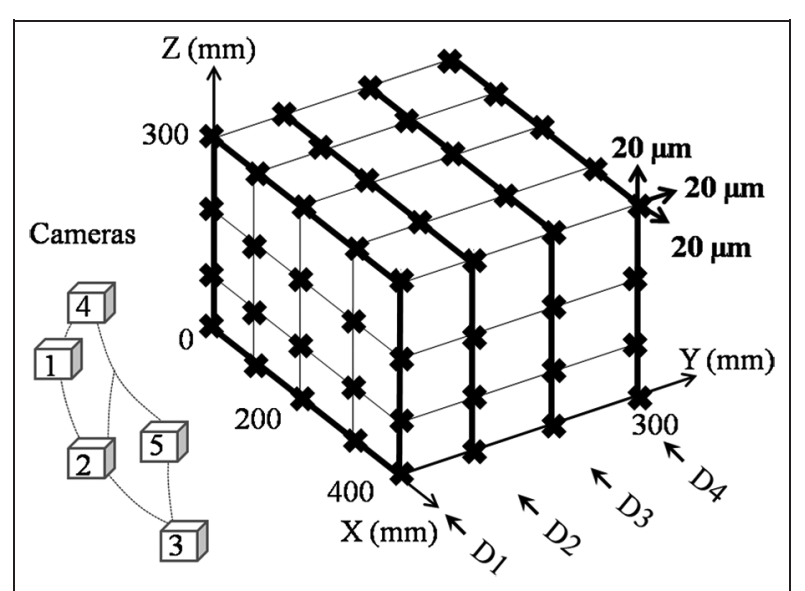

Figure 2. Demonstration of the 80 positions within the sampling volume $\left(400 \times 300 \times 300 \mathrm{~mm}^{3}\right)$. ' $*$ ' denotes the different positions. DI, D2, D3 and D4 are the different camera distance at $0,100 \mathrm{~mm}, 200 \mathrm{~mm}$ and $300 \mathrm{~mm}$ of $y$ axis, respectively. Bold lines: the vertical planes at different camera distances.

accurate measurements. A previous study has indicated that the resolution and system repeatability were affected by the random noise level. ${ }^{15}$ So, the markers coordinates, under static condition, was recorded to evaluate the random noise level in proposed system.

\section{Data processing}

Raw trajectory data of the markers were further processed with MATLAB (The MathWorks, Inc. Version 7.9.0 R2009b).

Coordinate transformation. The Cartesian dimensions of the milling machine could deviate from the Cartesian dimensions of the optical system. Coordinate transformation was performed to compensate such mis-alignment.

System accuracy. In order to minimize the effect of noise, marker coordinates data over $1 \mathrm{~s}$ (100 frames) at each position were averaged. The system accuracy describes the closeness of the recorded displacement to the reference displacement of the milling machine $(20 \mu \mathrm{m})$. In order to assess the effects of different parameters (calibration procedure, cameras distance, marker size and axis) on the performance of the optical system, the absolute error between recorded displacement and the reference displacement of each measurement was calculated. The measurement accuracy was then expressed as the percentage error, which is given by

$$
\text { Percentage error }=\frac{\text { absolute error }}{\text { reference value }} \times 100 \%
$$

System precision. The precision describes the repeatability of the recorded value with the same set-up of system. With the repetitions of the measurements at each camera distance (Figure 2: D1, D2, D3 and D4), the standard deviation (SD) of the recorded value was calculated as the system precision.
Random noise. Noise level along different axis was expressed as the root mean square (RMS) amplitude about the mean value of the data under static capturing with $100 \mathrm{~Hz}$.

\section{Statistics}

Statistical analyses were performed using $\mathrm{R}$ statistic software (version 2.12.2, R Development Core Team, 2011) and Graphpad Prism statistical software (version 5.00, GraphPad software, Inc., La Jolla, CA). Threeway analysis of variance (ANOVA) was employed to examine the main and interaction effects of the four parameters mentioned above (calibration procedure: manual and automatic calibration; marker size: $\emptyset 3 \mathrm{~mm}$, $\varnothing 4 \mathrm{~mm}, \emptyset 8 \mathrm{~mm}$ and $\varnothing 10.5 \mathrm{~mm}$; camera distance: D1, D2, D3 and D4; axis: $X, Y$ and $Z$ ) on the accuracy. Data regarding the accuracy of the system are presented as mean $( \pm \mathrm{SD})$ of the absolute error.

As the reflection of the system precision (the SD of the repeated measurements), the homogeneity of the variances of the recorded movement (reference movement is $20 \mu \mathrm{m}$ ) were assessed by the Fligner-Killeen's test. Main and interaction effects of system factors on the precision were reported. Data regarding to the precision are presented as the $\pm \mathrm{SD}$ value. Statistical significance was accepted at $p \leqslant 0.05$.

\section{Results}

\section{System accuracy}

Calibration procedure. For the displacement of $20 \mu \mathrm{m}$, the effects of calibration procedures were assessed by the optical system over all 80 positions. Statistical analysis of the data showed no significant $(p=0.37)$ main effect of the calibration procedure on system accuracy (Figure 3). However, significant main effects of camera distance $(p<0.001)$ and axis $(p<0.001)$ were found on the system accuracy. Moreover, an interaction effect $(p<0.001)$ between camera distance and axis was indicated. A greater error in the $Y$ axis was encountered for camera distance D3 and D4 than for D1 $(p<0.001)$.

Camera distance, marker size and axis. Statistical analysis of the data showed significant $(p<0.001)$ main effects of the camera distance and axis on system accuracy, respectively. System accuracy reduced (the absolute error increased) significantly along the $Y$ axis $(p=0.04)$. Furthermore, two-way interactions were found between camera distance and axis $(p<0.001)$, maker size and axis $(p=0.03)$, respectively. At camera distance D3, larger absolute errors in the $Y$ axis was associated with the usage of larger markers $(\varnothing 10.5 \mathrm{~mm})$ as compared with smaller markers $(\varnothing 3 \mathrm{~mm}, \varnothing 4 \mathrm{~mm}$ and $\emptyset 8 \mathrm{~mm})(p=0.02$, Figure 4). For all the markers, larger absolute errors in the $Y$ axis than the other axes were found at camera distances D2, D3 and D4 $(p<0.001$ versus $p=0.17$ at D1). 


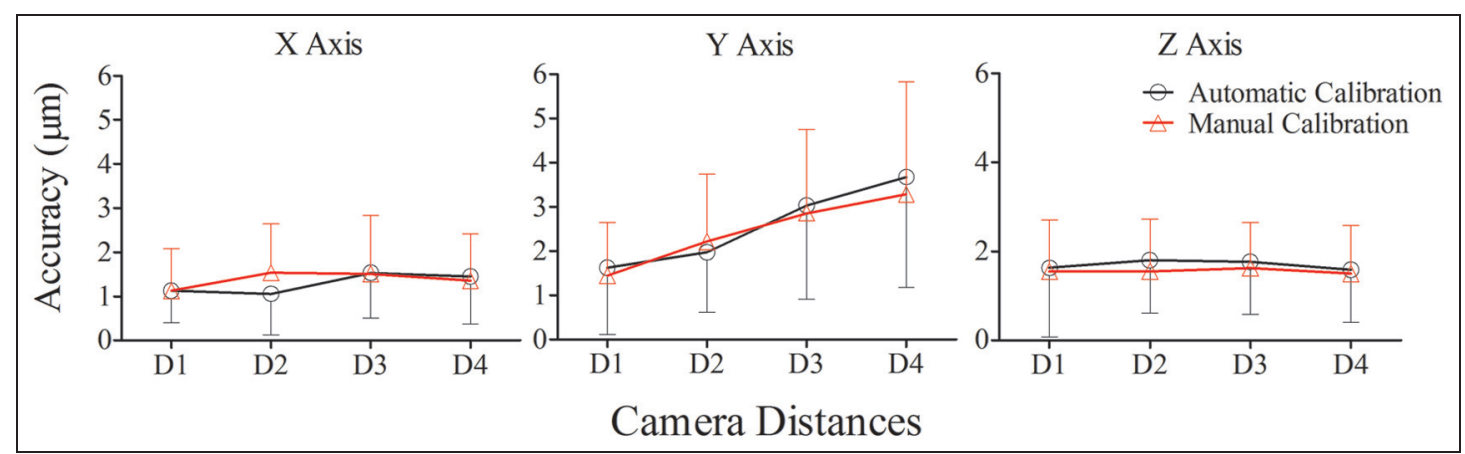

Figure 3. The system accuracy in the $X, Y$ and $Z$ axis with the automatic and manual calibration procedures, respectively. No significant difference on absolute error was found with two calibration procedures $(p=0.37)$. With both of the calibration procedures, the absolute error in the $Y$ axis increased with the increase of camera distance, especially in camera distance D3 and D4 $(p<0.00 \mathrm{I})$.

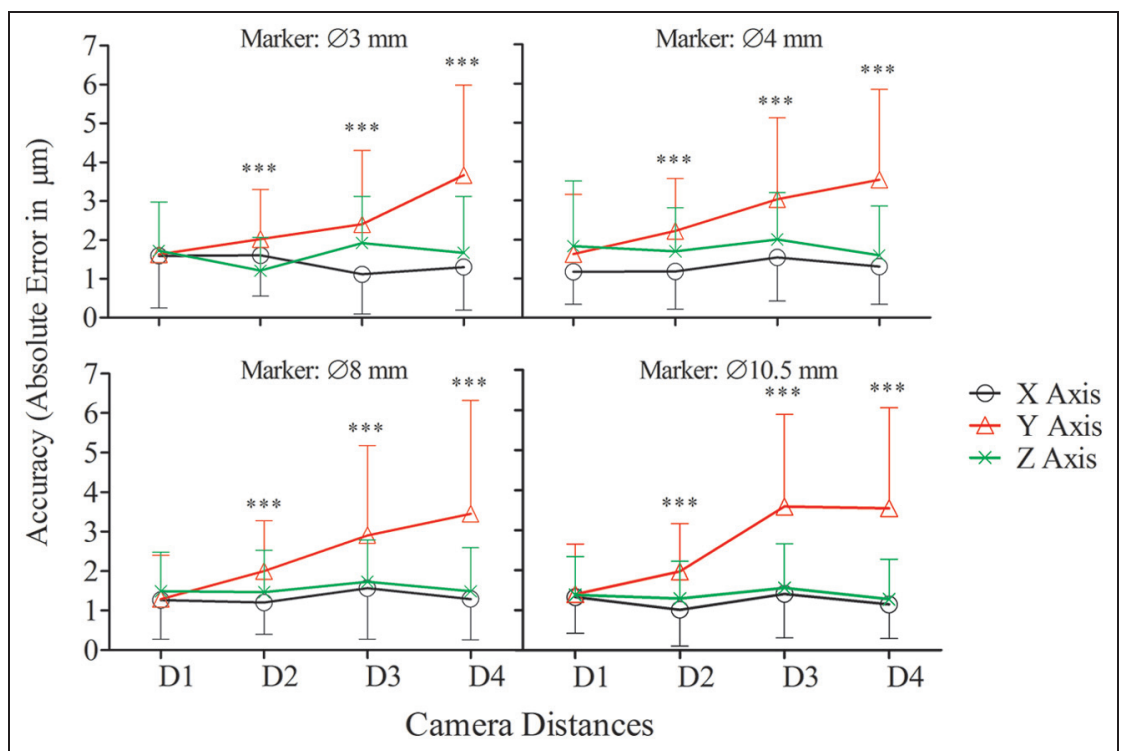

Figure 4. The effect of different parameters on the accuracy of the optical system. For all the markers, larger absolute error was induced with the increase of the camera distance in Yaxis at camera distance D2, D3 and D4 (***: $p<0.001)$. At the cameras distance D3, lager marker $(\varnothing 10.5 \mathrm{~mm})$ brought more absolute error than the other markers $(p=0.02)$.

By contrast, no significant main effect of marker size was found $(p=0.22)$. The statistical results indicated no other significant interaction effects, including threeway interactions among camera distance, maker size and axis $(p=0.16)$.

When averaged over all axes, the accuracy ranged $1.2-1.8 \mu \mathrm{m}(6 \%-9 \%)$ at $\mathrm{D} 1,1.0-2.2 \mu \mathrm{m}(5 \%-11 \%)$ at $\mathrm{D} 2,1.1-3.6 \mu \mathrm{m}(5.5 \%-18 \%)$ at D3 and $1.2-3.7 \mu \mathrm{m}$ $(6 \%-18.5 \%)$ at $\mathrm{D} 4$, respectively.

\section{System precision}

Calibration procedure. Both calibration procedures yielded comparable results with regards to system precision ( $p=0.65$, Figure 5). There were, however, main effects of camera distance at D2, D3 and D4, and axis in the $Y$ axis, demonstrating larger (worse) precision, as compared the other distances $(p<0.001)$ and axes $(p<0.001)$.

Camera distance, marker size and axis. Main effects of the three parameters (camera distance, marker size and axis) on system precision indicated the major differences in axes $(p<0.001)$ and camera distance $(p$ $<0.001)$, but only minor differences in marker size $(p=0.18$, Figure 6$)$.

All the different camera distances, except D1, were associated with a larger precision error along the $Y$ axis $(p<0.001, p=0.96$ at D1). At camera distance D3, the marker with $\varnothing 10.5 \mathrm{~mm}$ induced larger precision values than $\varnothing 3 \mathrm{~mm}$ marker in the $Y$ axis $(p=0.02$, Figure 6). No other interactions were found among camera distance, marker size and axis. For all the axes, 


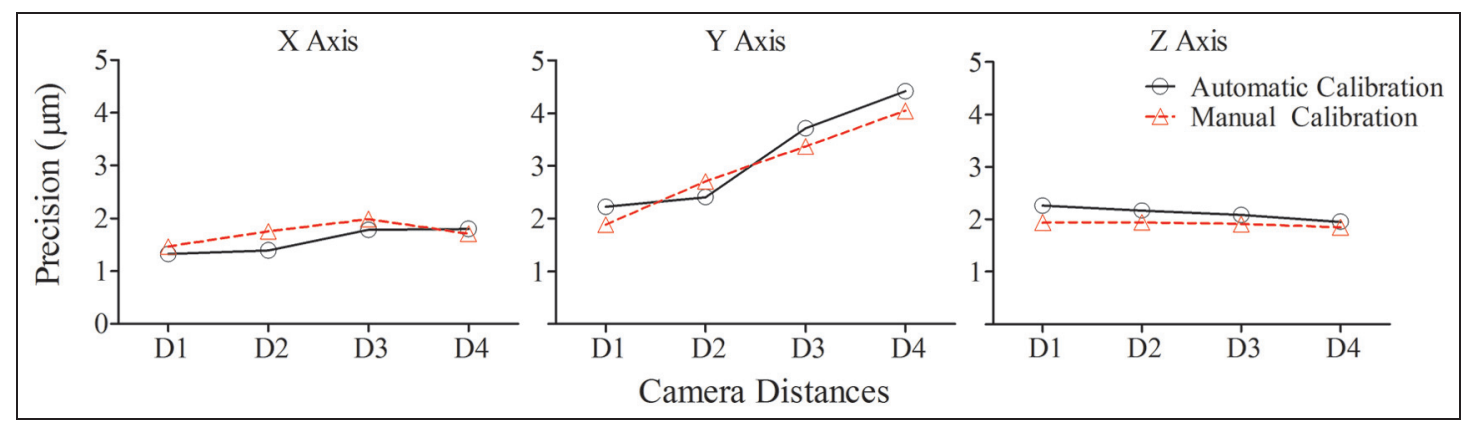

Figure 5. The precision at all 80 positions along the $X, Y$ and $Z$ axis with both calibration procedures. Similar to the results of absolute error, no significant difference in precision was found between the two calibration procedures $(p=0.65)$. With both the calibration procedures, the precision in the Yaxis increased with increasing camera distance $(p<0.00 \mathrm{I})$.

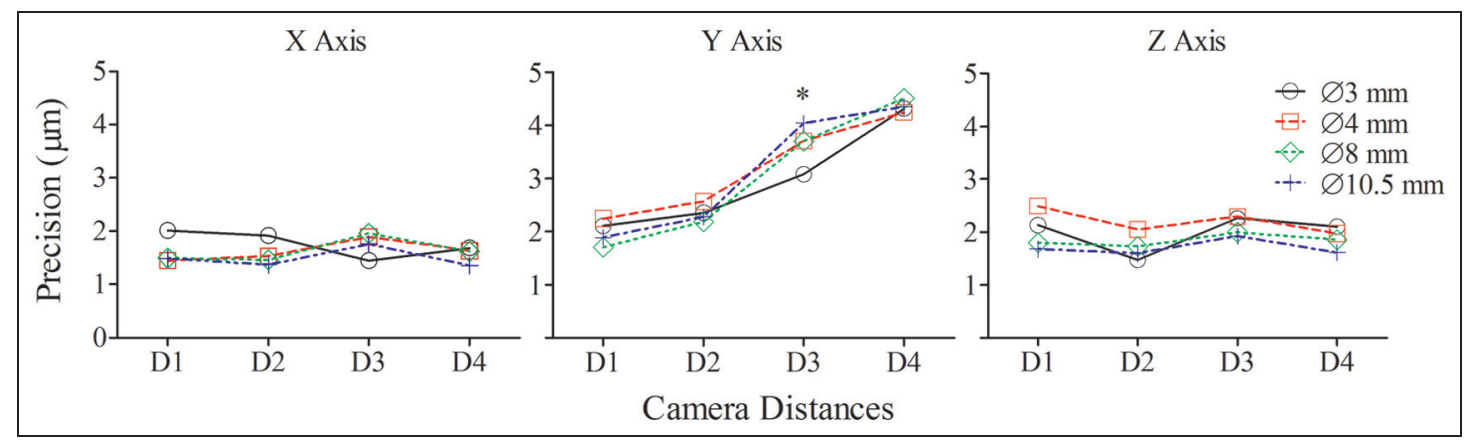

Figure 6. The effects of different camera distances, marker size and axis on the precision of the optical system. The precision value increased with the increase of the camera distance in the $Y$ axis. In the other axes, no effects of camera distance and marker size on the system precision were found. *: comparison of precision between $3 \mathrm{~mm}$ marker and $10.5 \mathrm{~mm}$ marker in the $Y$ axis at distance D3. $*: p<0.05$.

precision ranged $1.5-2.5 \mu \mathrm{m}$ at camera distance $\mathrm{D} 1$, $1.4-2.6 \mu \mathrm{m}$ at D2, $1.4-4.0 \mu \mathrm{m}$ at D3 and $1.4-4.5 \mu \mathrm{m}$ at $\mathrm{D} 4$, respectively.

\section{Random noise}

The system random noise (RMS amplitude) was $1.9 \mu \mathrm{m}$, $4.0 \mu \mathrm{m}$ and $3.0 \mu \mathrm{m}$ in $X, Y$ and $Z$ axis, respectively.

\section{Discussion and conclusions}

This study assessed the performance of an optical system for measuring small amplitude movements in a relative large volume. The effects of the calibration procedure, retro-reflective marker size, camera distance and axis upon system accuracy and precision have been evaluated. From the results of this study, it seems prudent to suggest that $20 \mu \mathrm{m}$ movements could be measured with acceptable accuracy and precision within a volume of $400 \times 300 \times 300 \mathrm{~mm}^{3}$, which means the system resolution was at least $20 \mu \mathrm{m}$ in this volume.

\section{Large volume}

Previous studies have successfully tracked the minute motion with high resolution, accuracy and precision, but only in a relatively small volume. ${ }^{14,15,18,19}$ The system information and results from previous studies are summarized in Table 1. Thus, compared with most previous studies, a better resolution (at least $20 \mu \mathrm{m}$ ), better accuracy and precision have been achieved by the presented approach, despite the fact that a larger volume has been used. It is evident that this improvement in system performance is mostly attributable to the higher resolution of the cameras (4 Megapixel), even though some parameters in the system configuration will probably also have contributions. More importantly, for most of the cases, the volume evaluated in this study covers the motion of the tibia during a stance phase of gait. The results suggest that the trajectory of the markers can be recorded in this volume with high accuracy and precision. Accordingly, the tibia deformation can be calculated with the same accuracy and precision if the markers are affixed to the tibia.

\section{Calibration procedure, camera distance and marker size}

Unlike the observations in the study of Windolf et al., ${ }^{14}$ calibration procedure did not affect the system performance in our study. Our results, therefore, imply that systematic error of this optical system, which are 
mostly induced by the calibration procedure, was very low and did not go to such an extent as to affect the system accuracy. In keeping with the conclusion of the study by Maletsky et al., ${ }^{18}$ increasing the camera distance increased the measured error and decreased the precision of the system along the main $Y$ axis. In some contrast with the findings from Windolf et al., ${ }^{14}$ greater marker size did not improve accuracy and precision over smaller markers, especially in the $Y$ axis. This finding can be explained as follows: Although more pixels are available with larger markers, that does imply an improvement for marker location precision, as more artifacts during the calculation of the marker center might be introduced while the marker moves further from cameras or the surface of the markers is not perfectly smooth. The other factors, such as different camera set-up, cameras numbers, marker quality (roundness and reflective quality) and surrounding environment condition, might also affect the performance of the optical system, as has been partly demonstrated in the past. ${ }^{14,17}$

\section{Why is the error enhanced in the $Y$ axis?}

At 80 positions of the measured volume, accuracy and precision have been evaluated in three orthogonal directions ( $X, Y$ and $Z$ axis). The results suggested that the accuracy and precision was greater in the $X$ and $Z$ axis than that in the $Y$ axis at two greatest camera distances (D3 and D4). A similar tendency was found when different sizes of markers were used. Interestingly, similar observations have been made in the study of Liu et al. ${ }^{15}$ and Windolf et al. ${ }^{14}$ as well. The likely explanation for these effects is that the number of pixels available from each marker diminishes when the markers are remote from the cameras. A reduction in pixel number is bound to enhance the error when calculating the central points of the marker with the gray scale reconstruction and circle fitting techniques. Oppositely, movement in the $X$ and $Z$ axis does not affect the pixel number, and it did accordingly not induce as large error as in $Y$ axis.

\section{Is the proposed approach good enough to serve its purpose?}

Owing to the irregular shape of long bones, axial deformation, shear, bending, torsion and their combination will occur on them under different loading condition. The traditional methods for measuring strains, e.g. strain gages, is not suitable for describing bone-segment deformation in three dimensions. ${ }^{5}$

As mentioned above, the purpose of this study was to establish an optical method and explore its application potential for in vivo bone-segment deformation measurements. For humans, the feasible anatomical sites for such measurements are limited. To date, most in vivo bone strain studies in humans have focused on the tibia. Results from these studies have shown that a 
peak principal tibia strain is approximately $1200 \mu \varepsilon$ under the conditions of daily normal activity, ${ }^{5,6,20}$ and that peak principal strains $>3000 \mu \varepsilon$ can be observed during more vigorous activities. ${ }^{21}$ However, in these studies, bone strains were measured by traditional strain gage approaches. With these technical set-ups, strains were available over a very limited surface area, typically a few square millimeters. To our knowledge, no methods are available to record the tibia-segment deformation in vivo so far, owing to the invasiveness and technical issues during the measurements. ${ }^{5}$

Based on the rough calculations mentioned in the 'Introduction' section and the results from this study, at least $20 \mu \mathrm{m}$ resolution, high accuracy and precision in the defined volume demonstrates that the present approach has the ability to record in vivo tibia deformation in humans.

Moreover, as indicated in a recent review article about in vivo bone strain measurements, ${ }^{5}$ no information is available in humans regarding the possible bending and torsion deformation in vivo. During the past decades, intracortical pins or screws with marker clusters were used in numerous studies to measure 3D kinematics of the tibiofemoral ${ }^{22}$ and the patellofemoral joint, ${ }^{23}$ as well as the foot bones. ${ }^{24}$ The relative bone motion in six degrees of freedom (three rotations and three translations) can be clearly described, most commonly, in the anatomical coordinate systems. ${ }^{25}$ For example, based on the Cardan-Eular angle, the flexion-extension, abduction-adduction, internal-external knee rotation and the translation can be calculated in the knee joint.

During kinematics studies, the bone segments were usually considered to be rigid, thus totally neglecting any bone deformation. However, the methodology to describe the flexion-extension, abduction-adduction,

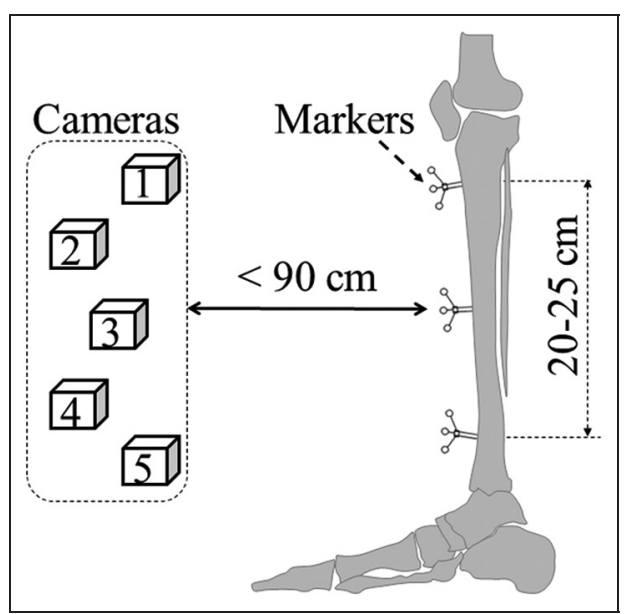

Figure 7. The recommended configuration for in vivo tibia deformation recording in humans. First, the marker clusters (at least three markers in one cluster) with small markers (Ø3$\emptyset 8 \mathrm{~mm})$ and close camera distance $(<90 \mathrm{~cm})$ should be used. In addition, the movement of the tibia along the optical axis of camera lens (Yaxis in this study) should be minimized as little as possible. internal-external rotation and the translation can still be adopted to refer to the bending, torsion and axial deformation of bone. So, the following set-up (Figure 7) is recommended to be used in tibia-segment deformation recording in vivo. Few bone pins or screws with retro-reflective marker clusters (three marker clusters with non-collinear markers on each cluster in Figure 7) could be inserted into the anterior-medial aspect of proximal, mid-shaft and distal tibia. The optical system and configuration proposed in this study then could be adopted to capture the coordinates of the markers during one step of the different locomotive activities, such as gait. Whatever the type of bone deformation is, axial, shear or torsion, all of them are able to be expressed by the relative movement between marker clusters and calculated based on the coordinates data of the markers.

\section{Recommendation for the application of the proposed method in bone-segment deformation in vivo measurements}

According to the results from this study and the proposed set-up of the markers in human tibia in Figure 7 , close camera positioning (camera distance: D1, $<90 \mathrm{~cm})$ and reducing marker size $(\varnothing 3-\varnothing 8 \mathrm{~mm})$ seems to be the best choice to improve the performance of this optical system and realize in vivo tibia-segment deformation recording with high accuracy and precision. Furthermore, the movement of the markers in the direction of cameras distance should be avoided, as much as the subjects can, during different exercises.

Concerning the detailed technically issues of applying the proposed method in tibia-segment deformation in vivo measurements, the pain induced by the screw insertion should be minimized by using thin bone screws. The invasiveness inflicted upon the subjects will be reduced with bone pins, as the periosteum will almost not be severed. In addition, a skin incision with appropriate length at the site of the bone screw insertions should be made to avoid the contact between soft tissues with bone screws during the exercises.

\section{Comparison between the proposed method and the existing method}

As the gold standard for measuring mechanical strain in engineering applications, strain gage methods have been often used in in vivo bone strain measurements in the past. However, the credibility of the strain gage approach is under debate owing to the uncontrollable of the bonding quality on the bone surface. This means that the so call 'gold standard' method might not suit in vivo bone strain measurements perfectly because of the potential poor bonding quality. Comparatively, the proposed method overcomes several drawbacks of the traditional strain gages method. First, it will provide the tibia-segment deformation information that has 
never been observed in vivo before. Integrating with anatomical coordinate system or more advanced method, such as finite element model, 3D in vivo tibia strain distribution will then be able to be calculated. Second, it will make it possible to explore to which extent bending and torsion are occurring in the human tibia. As mentioned above, on top of the previously mentioned novel possibilities by our proposed method, the strain detection limit of this new approach is acceptable for the in vivo bone-segment deformation recording. Based on different principals with the proposed method, several other optical methods, such as DIC ${ }^{9-11}$ and machine vision photogrammetry (DISMAP), ${ }^{26}$ have been explored to measure bone strains as well. Unquestionably, these methods have the ability of obtaining high-resolution strain distribution on certain areas of bone surface. However, so far, it is apparently difficult for these optical methods to measure the in vivo bone strains owing to its specific requirement of bone surface preparation and exposure to the cameras. This will probably be the main limitation for the application of the above two optical methods in the future of bone strain in vivo measurements.

\section{Conclusion}

To conclude, the performance of the proposed method, based on a motion capture system, is capable of recording the displacement of $20 \mu \mathrm{m}$ with high accuracy and precision in a relative large volume of $400 \times 300 \times 300$ $\mathrm{mm}^{3}$. Therefore, the results suggest that the proposed method has sufficient application potential in tibiasegment deformation in vivo measurements in the future.

\section{Funding}

This research received no specific grant from any funding agency in the public, commercial, or not-for-profit sectors.

\section{Acknowledgments}

We would like to thank Hans-Martin Küsel-Feldker and Thomas Förster at the Institute of Biomechanics and Orthopaedics, German Sport University Cologne, Germany, for operating the milling machine and numerous discussions about the ideas in this study. Thanks also to Michel Ducos for the discussion regarding to statistics. Peng-Fei Yang acknowledges his scholarship by the China Scholarship Council (CSC No.: 2009629013).

\section{Conflict of interest}

The Authors declare that there is no conflict of interest.

\section{References}

1 Duncan RL and Turner $\mathrm{CH}$. Mechanotransduction and the functional response of bone to mechanical strain. Calcif Tissue Int 1995; 57(5): 344-358.
2 Forwood MR and Turner CH. Skeletal adaptations to mechanical usage: results from tibial loading studies in rats. Bone 1995; 17(4 Suppl); 197S-205S.

3 Turner $\mathrm{CH}$, Owan I and Takano Y. Mechanotransduction in bone: role of strain rate. Am J Physiol 1995; 269(3 Pt 1): E438-442.

4 Mosley JR and Lanyon LE. Strain rate as a controlling influence on adaptive modeling in response to dynamic loading of the ulna in growing male rats. Bone 1998; 23(4): 313-318.

5 Yang PF, Bruggemann GP and Rittweger J. What do we currently know from in vivo bone strain measurements in humans? J Musculoskelet Neuronal Interact 2011; 11(1): 8-20.

6 Burr DB, Milgrom C, Fyhrie D, et al. In vivo measurement of human tibial strains during vigorous activity. Bone 1996; 18(5): 405-410.

7 Ekenman I, Halvorsen K, Westblad P, et al. The reliability and validity of an instrumented staple system for in vivo measurement of local bone deformation. An in vitro study. Scand J Med Sci Sports 1998; 8(3): 172-176.

8 Milgrom C, Finestone A, Hamel A, et al. A comparison of bone strain measurements at anatomically relevant sites using surface gauges versus strain gauged bone staples. J Biomech 2004; 37(6): 947-952.

9 Yang L, Zhang P, Liu S, et al. Measurement of strain distributions in mouse femora with 3D-digital speckle pattern interferometry. Opt Lasers Eng 2007; 45(8): 843-851.

10 Sztefek P, Vanleene M, Olsson R, et al. Using digital image correlation to determine bone surface strains during loading and after adaptation of the mouse tibia. $J$ Biomech 2010; 43(4): 599-605.

11 Zauel R, Yeni YN, Bay BK, et al. Comparison of the linear finite element prediction of deformation and strain of human cancellous bone to $3 \mathrm{D}$ digital volume correlation measurements. J Biomech Eng 2006: 128(1): 1-6.

12 Matsuyama J, Ohnishi I, Sakai R, et al. A new method for measurement of bone deformation by echo tracking. Med Engng Phys 2006; 28(6): 588-595.

13 Abdel-Aziz YI and Karara HM. Direct linear transformation from comparator coordinates into object space coordinates in close range photogrammetry. In: Proceedings of the symposium on close-range photogrammetry, Falls Church, VA: American Society of Photogrammetry, 1971.

14 Windolf M, Gotzen N and Morlock M. Systematic accuracy and precision analysis of video motion capturing systems - exemplified on the Vicon-460 system. $J$ Biomech 2008; 41(12): 2776-2780.

15 Liu H, Holt C and Evans S. Accuracy and repeatability of an optical motion analysis system for measuring small deformations of biological tissues. $J$ Biomech 2007; 40(1): 210-214.

16 Milgrom C, Finestone A, Levi Y, et al. Do high impact exercises produce higher tibial strains than running? $\mathrm{Br} J$ Sports Med 2000; 34(3): 195-199.

17 Nigg BM and Herzog W (eds). Biomechanics of the musculo-skeletal system. West Sussex: Wiley and Sons Ltd, 2007.

18 Maletsky LP, Sun J and Morton NA. Accuracy of an optical active-marker system to track the relative motion of rigid bodies. $J$ Biomech 2007; 40(3): 682-685.

19 Schmidt J, Berg DR and Ploeg H-L. Precision, repeatability and accuracy of Optotrak optical motion tracking systems. Int J Experl Compl Biomech 2009; 1: 114-127. 
20 Milgrom C, Miligram M, Simkin A, et al. A home exercise program for tibial bone strengthening based on in vivo strain measurements. Am J Phys Med Rehabil 2001; 80(6): 433-438.

21 Milgrom C, Simkin A, Eldad A, et al. Using bone's adaptation ability to lower the incidence of stress fractures. $\mathrm{Am}$ $J$ Sports Med 2000; 28(2): 245-251.

22 Ramsey DK, Wretenberg PF, Benoit DL, et al. Methodological concerns using intra-cortical pins to measure tibiofemoral kinematics. Knee Surg, Sports Traumat, Arthros 2003; 11(5): 344-349.

23 Ramsey DK and Wretenberg PF. Biomechanics of the knee: methodological considerations in the in vivo kinematic analysis of the tibiofemoral and patellofemoral joint. Clin Biomech (Bristol, Avon) 1999; 14(9): 595-611.

24 Nester C, Jones RK, Liu A, et al. Foot kinematics during walking measured using bone and surface mounted markers. J Biomech 2007; 40(15): 3412-3423.

25 Grood ES and Suntay WJ. A joint coordinate system for the clinical description of three-dimensional motions: application to the knee. J Biomech Eng 1983; 105(2): $136-144$.

26 Kim DG, Brunski IB and Nicolella DP. Microstrain fields for cortical bone in uniaxial tension: optical analysis method. Proc IMechE, Part H: J Engineering in Medicine 2005; 219(2): 119-128. 